\title{
Ségurant ou Le Chevalier au dragon, Tome I, Version cardinale, Édition critique par E. ARIOLI
}

\author{
Maria Colombo Timelli
}

\section{OpenEdition}

\section{Journals}

Édition électronique

URL : https://journals.openedition.org/studifrancesi/32573

DOI : 10.4000/studifrancesi.32573

ISSN : 2427-5856

Éditeur

Rosenberg \& Sellier

Édition imprimée

Date de publication : 1 août 2020

Pagination : $375-376$

ISSN : 0039-2944

\section{Référence électronique}

Maria Colombo Timelli, « Ségurant ou Le Chevalier au dragon, Tome I, Version cardinale, Édition critique par E. ARIOLI », Studi Francesi [En ligne], 191 (LXIV | II) | 2020, mis en ligne le 01 septembre 2020, consulté le 18 septembre 2021. URL : http://journals.openedition.org/studifrancesi/32573 ; DOI : https://doi.org/10.4000/studifrancesi.32573

Ce document a été généré automatiquement le 18 septembre 2021.

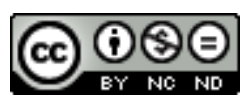

Studi Francesi è distribuita con Licenza Creative Commons Attribuzione - Non commerciale - Non opere derivate 4.0 Internazionale. 


\title{
Ségurant ou Le Chevalier au dragon, Tome I, Version cardinale, Édition critique par E. ARIOLI
}

\author{
Maria Colombo Timelli
}

\section{RÉFÉRENCE}

Ségurant ou Le Chevalier au dragon, Tome I, Version cardinale, Édition critique par E.

ARIOLI, Paris, Honoré Champion, 2019, «Classiques français du Moyen Âge» 188, 402 pp.

1 Un «roman de Ségurant», disons-le tout de suite, n'existe pas (ou plus), le texte reconstruit par E. Arioli consistant de fait en une collection d'épisodes conservés dans des manuscrits relevant de la tradition arthurienne: Prophéties de Merlin, Guiron le Courtois, Compilation de Rusticien de Pise pour la plupart. Il s'agit ici de la «Version cardinale», la plus ancienne (1240-1279): transmise par le manuscrit Arsenal 5229 (fin $\mathrm{XIV}^{\mathrm{e}}$-début XV ${ }^{\mathrm{e}}$ siècle), elle compte 39 épisodes entremêlés aux Prophéties de Merlin.

2 L'Introduction (pp. 11-77), aussi claire qu'informée, fournit au lecteur tous les éléments pour situer cette version au sein d'une tradition enchevêtrée dont E. Arioli a amplement rendu compte dans le tome 45 de l'Histoire littéraire de la France (SF 181, 2017, p. 128). L'étude littéraire porte en particulier sur les liens entre Ségurant et la tradition arthurienne (Tristan en prose, Lancelot en prose: on verra à ce propos les «renvois explicites à d'autres romans» réunis et commentés dans l'Annexe, pp. 331-337); le manuscrit de l'Arsenal fait l'objet d'une description minutieuse, qui prend en compte outre les aspects matériels - son histoire et sa composition interne; les Remarques sur la langue signalent les phénomènes les plus voyants, parmi lesquels quelques traces d'italianismes (on pourrait ajouter à ceux-ci l'emploi de nies / nepveu pour 'petit-fils': f. 50rb, p. 110 de l'édition, f. 50vb, p. 111). Les Principes d'édition sont aussi fournis dans le détail. Quelques-uns des épisodes édités dans ce volume se lisant aussi dans d'autres 
manuscrits, on en trouvera la liste dans un Tableau synthétique très bien venu. Bibliographie essentielle aux pp. 79-84.

Le manuscrit (numérisé en couleur dans Gallica) ne présente pas de difficulté de lecture, et le texte est édité avec soin. Je me permets néanmoins quelques remarques: p. 99, f. 49vb, je lirais «Et quant la nouvelle fu seue por tos, sachés veraiement que cil que fu illec abatu s'i (et non pas si) tient a gari»; de même p. 194, f. 93ra, «par aventure s'i (au lieu de si) se combatoit puys aprés la jouste...»; p. 229, f. 108rb: «et quant il vit Lamorat retourner au renc appereillé de jouste...» (j'aurais transcrit jousté, graphie attestée ailleurs pour les infinitifs du premier groupe, comme le souligne E.A. p. 41). L'équivalence graphique $s / c$ étant bien signalée dans le commentaire linguistique (p. 43), je ne serais pas intervenue pour corriger «Biaux fils, de ce [se dans le ms] ne soiez point adoulez...» (p. 115, f. 52vb), à plus forte raison que ce pour se est conservé ailleurs («nul chevalier ne s'i venoit esprouver, ce il n'estoit bien de hault afaire», p. 151, f. 73ra; «se estoit...», p. 194, f. 93ra). Pour ce qui est de la soudure / division des mots, j'aurais différencié pourquoi (adv. interr.) et pour quoi (préposition + pronom relatif), par exemple p.109, f. 50ra-b: «Seguranz tis filz sera preux et hardiz oultre mesure [...] et en a bien monstree droite esprouve, pour quoy je vueil le adouber a chevalier»; ou p. 210, f. 100ra: «cil chevaliers sont de hault lignage, por quoy il dit qu'il demourera avec eulx». La ponctuation devrait être parfois rectifiée ou complétée: p. 109, f. 50ra, «il estoit tant hardiz tant vistes et tant preux...» (ajouter une virgule après hardiz); p. 221, f. 105va, «Quant cele grant navie fut arrivee [...] et li barons et li chevaliers commencent a yssir des nez. Lors peussiez veoir...» (remplacer le point par une virgule); p. 222, f. 106rb, les prises de parole de Guenièvre et de la Dame de Malehaut doivent être différemment réparties pour que le dialogue garde son sens: «Et quoy m'en loez vous a faire, dame? fait la Dame de Malohaut. Je voy bien...» (et non pas: «Et quoy m'en loez vous a faire? - Dame, fait la Dame de Malohaut, je voy bien...»). Dans l'ensemble, il ne s'agit cependant que de retouches minimes, qui n'enlèvent rien à la valeur de l'édition.

4 Le volume comprend aussi un choix des variantes pour les trois épisodes transmis par d'autres manuscrits (pp.339-355), un Index onomastique (pp.357-366), un Index toponymique (pp. 367-372) et le Glossaire (pp. 373-397); les critères pour l'établissement de celui-ci n'ayant pas été explicités, je signalerais qu'il contient des mots aussi familiers au lecteur que acoler ou baillier, mais exclut quelques locutions intéressantes, par exemple tenir nuit («Segurans tint nuit devant l'autel a grant devocion», p.111, f. 50vb) ou a cent doubles («fut elle plus regardee a cent doubles, quant elle y vint», f. 103ra, p. 218; «elle prise orendroit la pierre a cent doubles plus qu'elle ne la prisoit devant», f. 103va-b, p. 220).

5 La lecture de Ségurant s'avère très agréable, le lecteur n'ayant pas le sentiment - grâce aussi au commentaire d'E. Arioli - d'avoir à faire à des fragments décousus; sur le plan textuel le «roman» ne présente pas les défauts communs à d'autres prosateurs, ou à d'autres copistes, de la fin du Moyen Âge, et ce malgré la répétition de certains clichés stylistiques: soulignons par exemple le retour de la foudre en tant que comparant pour la rapidité ou le bruit dans les mêlées («Aussi l'aloient tuit autresi eschivant com ce feust la foudre qu'ilz veissent venir», p. 113; «tuit s'enfuient devant lui aussi com se la foudre les chaçast», p. 114; «ilz fuyoient devant luy ainsi com se la fouldre les chaçast» encore p. 168; «et lessent courre par si grant force que ce semble fouldre droitement», p. 153; «Fuyez, veez les fouldres venir!», p. 170; bruyant / bruiant comme la fouldre se lit aux pp. 152, 153, 
179, 229 et j'en passe); on peut donc espérer que cette édition suscitera l'intérêt des médiévistes, confrontés à un texte inconnu, tout au moins sous la forme que lui a donnée E.A. Il ne reste maintenant qu'à attendre la publication de l'étude destinée à les accompagner, à paraître dans la «Nouvelle Bibliothèque du Moyen Âge», annoncée ici p. 10 . 